\title{
MiR-146a Regulates Inflammatory Infiltration by Macrophages in Polymyositis/Dermatomyositis by Targeting TRAF6 and Affecting IL-17/ ICAM-1 Pathway
}

\author{
Yuanqin Yin ${ }^{a}$ Fei Li ${ }^{b}$ Jing Shic Songlin Lid Jingjing Caie Youhong Jianga \\ ${ }^{a}$ Cancer Institute, First Affiliated Hospital, China Medical University, Shenyang, ${ }^{b}$ Department of Internal \\ Medicine, Cancer Hospital of China Medical University, Liaoning Cancer Hospital \& Institute, Shenyang, \\ 'Department of Obstetrics and Gynecology, Cancer Hospital of China Medical University, Liaoning \\ Cancer Hospital \& Institute, Shenyang, dDepartment of Respiratory Medicine, Tangshan Worker \\ Hospital, Tangshan, e'General Hospital of Fushun Mining Bureau, Fushun, China
}

\section{Key Words}

PM/DM • TRAF6 $・$ IL-17 $・$ ICAM-1 $・$ Macrophage $・$ Inflammatory

\begin{abstract}
Background/Aims: The primary objective of this study was to investigate the role of miR-146a in inducing the inflammatory infiltration of macrophages in polymyositis/dermatomyositis (PM/DM) through targeting TNF receptor associated factor 6 (TRAF6), which may further down-regulate the Interleukin-17 (IL-17)/Intercellular Adhesion Molecule 1 (ICAM-1) pathway. Methods: Biopsies were collected from PM/DM patients and healthy volunteers. PM/DM model establishment and macrophage isolation were performed on Sprague Dawley (SD) rats. Model rats and macrophages were treated with anti-IL-17, anti-ICAM-1, miR-146a mimics, miR-146a inhibitors, and TRAF6 siRNAs. Serum creatine phosphokinase (S-CK) expression was assessed using double antibody sandwich enzyme-linked immunosorbent assay (ELISA) assay, and immunohistochemistry assay was performed to analyze CD163 expression in muscle samples. Furthermore, we used transwell assay to test cell migration; RT-PCR and western blot were carried out to determine the expression of miR-146a, TRAF6, IL-17, and ICAM-1. Results: The S-CK, TRAF6, IL-17 and ICAM-1 levels were higher in PM/DM patients compared with healthy controls and were down-regulated after the conventional treatment. Treatment with miR-146a mimics, anti-IL-17 and anti-ICAM-1 decreased the expression of IL-17 and ICAM-1, whereas miR-146a inhibitors exerted the opposite effects. The effects of miR-146a inhibitors were suppressed by treatment with TRAF6 siRNA. In addition, the luciferase reporter assay validated the targeting relationship between miR-146a and TRAF6. Conclusions: MiR-146a regulates inflammatory macrophage infiltration in PM/DM by targeting TRAF6 and affecting the IL-17/ICAM-1 pathway.
\end{abstract}

(C) 2016 The Author(s)

Published by S. Karger AG, Basel

Youhong Jiang

KARGER
Cancer Institute, First Affiliated Hospital, China Medical University, No. 155 Nanjing North Street, Heping District, Shenyang, Liaoning, 110001, (China)

Tel. +86 024-83232354, E-Mail jiangyouhong2000@aliyun.com 


\section{Cellular Physiology Cell Physiol Biochem 2016;40:486-498 \begin{tabular}{ll|l} 
DOI: 10.1159/000452563 25,2016 & $\begin{array}{l}\text { O) 2016 The Author(s). Published by S. Karger AG, Basel } \\
\text { www.karger.com/cpb }\end{array}$
\end{tabular} \\ Yin et al.: MiR-146a/TRAF6 Alters IL-17/ICAM-1 in Polymyositis}

\section{Introduction}

Polymyositis/dermatomyositis (PM/DM), which is characterized by meristic proximal muscle weakness and the elevation of muscle enzyme level, is an unusual idiopathic inflammatory myopathy [1]. The reported incidence is 1 in 250,000 to 1 in 130,000 per annum, and the prevalence of PM/DM is approximately 1 in 14,000 according to previous reports [2]. Researchers have begun to investigate the underlying pathology of PM/DM, which is related to abnormal autoimmunity regulation. Additionally, a combination of genetic polymorphisms and environmental factors appear to be relevant [1]. As a result, there is no evidence-based theory to determine the appropriate treatment for PM/DM, and physician experience and empirical data play key roles in treatment selection [3]. Furthermore, the timely diagnosis of PM is difficult because its symptoms are very similar to those of myositis [4]. Therefore, the identification of myositis in PM/DM will aid clinicians in determining the key risk factors that are critical for treatment selection, representing a breakthrough in clinical practice [3].

Interleukin-17 (IL-17), which is produced by T-helper cells, is a pro-inflammatory cytokine and induces TNF $\alpha$, IL-1b, IL-6, IL-23 and G-CSF [5]. Because IL-17 has also been detected in the sera of patients with various diseases, it has been linked to the development of various human diseases [6], including multiple sclerosis [7], systemic lupus erythematosus, allograft rejection, and asthma [8]. Intercellular Adhesion Molecule 1 (ICAM-1), also known as CD54, is a member of the immunoglobulin super family [9] and is expressed in both a soluble form and a membrane-bound form on the surface of several types of cells, including endothelial cells and immune cells; furthermore, ICAM-1 is induced by cytokines such as TNF- $\alpha$, interleukin- 1 and bacterial lipopolysaccharide [10]. ICAM-1 is detectable in the blood serum and cerebrospinal fluid when patients experience infections, autoimmune or proliferative diseases [11]. Recent evidence suggests the synergy of Interleukin-17 and IFN $\gamma$ or TNF $\alpha$ in promoting ICAM-1 expression in many inflammatory diseases [12]. Therefore, we suspected a significant role for IL-17-ICAM-1 in polymyositis.

MicroRNAs (miRNAs) are a family of small, endogenous non-coding RNAs and are involved in the regulation of multiple biological processes ranging from cell fate determination to signaling events at post-transcription level $[13,14]$. MiR-146a is a member of the miR-146 miRNA family and it shares the same seed sequence with miR-146b-5p. MiR-146a modulates innate as well as adaptive immunity [15] by negatively regulating TNF receptor-associated factor 6 (TRAF6) and IL-1 receptor-associated kinase 1 (IRAK1), two adapter proteins, both of which are important for pro-inflammatory signaling [16]. The upregulation of miR-146a downregulates IRAK1/TRAF6 expression, subsequently inhibiting $\mathrm{NF}-\kappa \mathrm{B}$ signaling and the expression of downstream targets proteins (ICAM-1, VCAM-1, IL-6 and PGE2) [17]. In peripheral blood mononuclear cells (PBMCs) and the synovium in rheumatoid arthritis patients, miR-146a is associated with the expression of IL-17 [5]. Therefore, we hypothesized that miR-146a is associated with IL-17/ICAM-1 pathway in polymyositis. The primary objective of this study was to investigate the role of miR-146a in inducing inflammatory infiltration of macrophages in PM/DM by targeting TRAF6, which may further up-regulate the IL-17/ICAM-1 pathway.

\section{Materials and Methods}

\section{Clinical samples}

Twenty patients (male : female $=6: 14$ ) with a medium age of 61 years old were diagnosed with polymyositis (PM) or dermatomyositis (DM) according to the criteria of Bohan and Peter [18] at the First Affiliated Hospital, China Medical University, between Mar 2014 and Oct 2014. All patients were treated with high dosages of glucocorticoids or other immunosuppressive substances, including methotrexate, cyclophosphamide, and azathioprine. Seven females and three males without clinical or histopathological signs of any muscle disease were included in the control group (medium age: 56 years old). The clinical 


\section{Cellular Physiology Cell Physiol Biochem 2016;40:486-498 \begin{tabular}{ll|l} 
DOI: 10.1159/000452563 25,2016 & $\begin{array}{l}\text { O } 2016 \text { The Author(s). Published by S. Karger AG, Basel } \\
\text { www.karger.com/cpb }\end{array}$ \\
and Biochemistry Published online: November
\end{tabular} \\ Yin et al.: MiR-146a/TRAF6 Alters IL-17/ICAM-1 in Polymyositis}

Table 1. Clinical data on patients and healthy controls. M, male; F, female; NA, not available; PM, polymyositis; DM, dermatomyositis; ANA, antinuclear antibody; Ro52, Sjoegren syndrome type A antigen; Ro60, Sjoegren syndrome antigen A2; Mi-2, chromodomain-helicase-DNA binding protein; SRP, signal recognition particle; Pred, prednisolone; MTX, methotrexate; AZA, azathioprine; s-CK, serum creatine phosphokinase; FI, functional index

\begin{tabular}{|c|c|c|c|c|c|c|c|c|c|c|c|}
\hline \multirow{2}{*}{ No. } & \multirow{2}{*}{ Sex } & \multirow{2}{*}{ Age } & \multirow{2}{*}{ Diagnosis } & \multirow{2}{*}{$\begin{array}{l}\text { Duration } \\
\text { months }\end{array}$} & \multirow{2}{*}{ Autoantibodies } & \multicolumn{3}{|c|}{ Biopsy I } & \multicolumn{3}{|c|}{ Biopsy II } \\
\hline & & & & & & Therapy & s-CK (IU/liter) & $\mathrm{FI}$ & Therapy & $\mathrm{s}$-CK (IU/liter) & $\mathrm{FI}$ \\
\hline 1 & $\mathrm{~F}$ & 65 & definite DM & 4 & ANA, Anti-R052 & no & 449 & 81 & Pred & 190 & 90 \\
\hline 2 & M & 67 & definite DM & 11 & ANA & no & 403 & 22 & Pred & 170 & 24 \\
\hline 3 & M & 62 & probable PM & 4 & ANA, Anti-RO52 & no & 428 & 28 & Pred & 188 & 36 \\
\hline 4 & $\mathrm{~F}$ & 63 & definite DM & 9 & ANA & no & 394 & 17 & Pred, MTX & 153 & 21 \\
\hline 5 & $\mathrm{~F}$ & 61 & definite PM & 5 & ANA & no & 399 & 47.5 & Pred & 212 & 58 \\
\hline 6 & $\mathrm{~F}$ & 60 & probable DM & 5 & Anti-Jo-1, Anti-Ro52 & no & 467 & 34.5 & Pred & 175 & 44 \\
\hline 7 & $\mathrm{~F}$ & 55 & definite DM & 6 & Negative & no & 468 & 28 & Pred & 167 & 39 \\
\hline 8 & $\mathrm{~F}$ & 44 & definite DM & 4 & ANA & no & 473 & 22 & Pred, MTX & 195 & 32 \\
\hline 9 & $\mathrm{~F}$ & 41 & probable PM & 4 & ANA, Anti-R052 & no & 382 & 50 & Pred & 222 & 53 \\
\hline 10 & $\mathrm{~F}$ & 68 & probable DM & 12 & ANA, Anti-R052, Anti- & no & 404 & 58 & Pred, MTX & 258 & 63 \\
\hline 11 & $\mathrm{~F}$ & 53 & definite DM & 7 & Negative & no & 425 & 54 & Pred, AZA & 196 & 58 \\
\hline 12 & $\mathrm{~F}$ & 47 & definite PM & 8 & ANA, Anti-Ro60 & no & 384 & 72.5 & Pred, AZA & 237 & 81 \\
\hline 13 & $\mathrm{~F}$ & 87 & definite DM & 5 & ANA & no & 373 & 53 & Pred, AZA & 182 & 63 \\
\hline 14 & $\mathrm{~F}$ & 71 & definite PM & 10 & ANA, Anti-R052, Anti-Ro60 & no & 413 & 60 & Pred, MTX & 238 & 67 \\
\hline 15 & M & 70 & probable DM & 7 & ANA & no & 374 & 71 & Pred, MTX & 216 & 80 \\
\hline 16 & M & 60 & probable PM & 9 & Anti-Mi-2 & no & 382 & 54 & Pred, AZA & 182 & 59 \\
\hline 17 & $\mathrm{~F}$ & 72 & definite PM & 10 & ANA, Anti-Ro60 & no & 445 & 48 & Pred, AZA & 176 & 58 \\
\hline 18 & $\mathrm{~F}$ & 48 & definite PM & 5 & ANA & no & 388 & 34.5 & Pred & 193 & 40 \\
\hline 19 & M & 58 & definite PM & 8 & Negative & no & 441 & 30.5 & Pred, AZA & 371 & 36 \\
\hline 20 & M & 71 & definite PM & 8 & ANA & no & 368 & 33 & Pred & 238 & 44 \\
\hline 21 & F & 58 & & & & & & & & 106 & \\
\hline 22 & $\mathrm{~F}$ & 47 & & & & & & & & 108 & \\
\hline 23 & $\mathrm{~F}$ & 64 & & & & & & & & 93 & \\
\hline 24 & $\mathrm{~F}$ & 61 & & & & & & & & 104 & \\
\hline 25 & M & 68 & & & & & & & & 87 & \\
\hline 26 & M & 42 & & & & & & & & 89 & \\
\hline 27 & F & 45 & & & & & & & & 85 & \\
\hline 28 & $\mathrm{~F}$ & 41 & & & & & & & & 111 & \\
\hline 29 & $\mathrm{~F}$ & 58 & & & & & & & & 92 & \\
\hline 30 & M & 52 & & & & & & & & 115 & \\
\hline
\end{tabular}

characteristics of the participants are presented in Table 1. Two biopsies were obtained from each patient: one was acquired before the treatment, and the other was obtained 8 months after treatment with glucocorticoids. The tissue samples collected in a semi-open assay were then frozen at $-80^{\circ} \mathrm{C}$ [19]. One clinical assessment consisted of the myositis functional index (FI), which measures performed repetition numbers in different muscle groups and was presented as a percentage of the max score of 64 . This study was reviewed and approved by the institutional ethics committee of the First Affiliated Hospital, China Medical University, according to the Helsinki Declaration. Informed consent was obtained from patients prior to enrollment.

\section{Animal model}

Forty male Sprague Dawley (SD) rats (weight: 225-350 g, age: 2-3 months) were obtained from China Medical University. Animal models of polymyositis were established by performing multiple abdominal injections of rabbit skeletal muscle homogenate as previously described [20]. Rats were randomly assigned to 4 experimental groups: the control group (treated with placebo operation), model group (established polymyositis model), anti-IL-17 group (polymyositis-model rats injected daily with $35 \mu \mathrm{Ci}$ anti-IL-17 monoclonal antibody ( $\mathrm{mAb}$ ) after the $14^{\text {th }}$ day post-operation), and the anti-ICAM-1 group (polymyositismodel rats injected daily with $35 \mu \mathrm{Ci}$ anti-ICAM-1 mAb after the $14^{\text {th }}$ day post-operation). All rats were checked and euthanized 4 weeks after the operation.

\section{Tests for serum creatine phosphokinase (S-CK), IL-17 and ICAM-1}

S-CK, IL-17 and ICAM-1 levels were assessed in blood samples extracted from each group by performing a double antibody sandwich enzyme-linked immunosorbent assay (ELISA) (R\&D Systems, Minneapolis, MN, USA) according to the kit's instruction.

\section{Cell culture adherence}

Peritoneal macrophages were isolated from SD rats. Phosphate buffered saline (PBS) containing 3\% Brewer's thioglycollate solution was injected into the peritoneal cavity of SD rats. Three days later, the peritoneal cavity was lavaged with PBS, and macrophage cells were harvested. After straining with 70 $\mu \mathrm{m}$ mesh and washing with ammonium-chloride-potassium (ACK) buffer, the cells were re-suspended in 


\section{Cellular Physiology Cell Physiol Biochem 2016;40:486-498 \begin{tabular}{ll|l} 
and Biochemistry Published online: November 25, 2016 & $\begin{array}{l}\text { ○ 2016 The Author(s). Published by S. Karger AG, Basel } \\
\text { www.karger.com/cpb }\end{array}$ \\
\hline
\end{tabular} \\ Yin et al.: MiR-146a/TRAF6 Alters IL-17/ICAM-1 in Polymyositis}

Roswell Park Memorial Institute medium (RPMI). After a $2 \mathrm{~h}$ culture period at $37^{\circ} \mathrm{C}$ in a $5 \% \mathrm{CO}_{2}$ incubator, the macrophage cells were isolated with PBS and maintained in RPMI. The macrophages were divided into 7 groups: the control group (cells without any treatment), the miR-146a mimics group (cells transfected with miR-146a mimics), the miR-146a inhibitors group (cells transfected with miR-146a inhibitors), the miR-146a inhibitors + TRAF6 siRNA group (cells transfected with miR-146a inhibitors and TRAF6 siRNA), the miR-146a mimics negative control group (NC mimics, cells transfected with miR-146a mimics negative control), the miR-146a inhibitors negative control group (NC inhibitors, cells transfected with miR-146a inhibitors negative control), and the siRNA negative control group (NC siRNA, cells transfected with siRNA for no specific sequence). Mimics, inhibitors, negative control mimics, negative control inhibitors and siRNAs were purchased from Santa Cruz Biotechnology (Santa Cruz, CA, USA) and transfected into cells using lipofectamine RNAiMAX (Invitrogen, Carlsbad, CA, USA) at the indicated concentrations.

\section{Immunohistochemistry}

Before the performance for histology analysis, muscle tissues were treated with $30 \%$ sucrose solution and $4 \%$ paraformaldehyde for perfusion. The frozen tissues were cut into slices, which were then applied with primary antibodies against CD163 (dilution 1:1000, BD Biosciences, San Jose, CA, USA). Samples were then incubated with horseradish peroxidase-conjugated (HRP-conjugated) goat anti-rabbit IgGs (1:800, Zhongshan Biology Company, Beijing, China), the secondary antibodies. After being visualized with diaminobenzidine (DAB, Sigma-Aldrich, St. Louis, MO, USA), the samples were hydrated, washed and analyzed by microscopy. In the negative control group, cells were treated with nonspecific IgGs (1:800, Zhongshan Biology Company, Beijing, China) to replace the primary antibodies.

\section{Luciferase activity assay}

The sequence of 3' untranslated region (UTR) of TRAF6 containing the miR-146a binding site was amplified by polymerase chain reaction (PCR) and cloned into the downstream site of the pGL3 luciferase vectors (Promega, Madison, WI, USA). The mutated binding site were obtained using the GeneTailor SiteDirected Mutagenesis System (Invitrogen, Carlsbad, CA, USA), and then the mutated 3' UTR was also cloned into the pGL3 luciferase vectors (relevant primer sequences are listed in Table 2). The macrophages were maintained in 96-well plates. Reporter vectors that carry TRAF6 3' UTR or mutated TRAF6 3' UTR or the control sequences were cotransfected with $200 \mathrm{ng}$ pGL3-control luciferase reporter and $10 \mathrm{ng}$ of pRLTK vectors (the internal control reporters). The assay used the Dual-Luciferase Reporter Assay System (Promega, Madison, WI, USA) with three repeated experiments according to the kit instructions. Cells were collected $48 \mathrm{~h}$ later, and relative luciferase activities were quantified.

\section{Transwell assay}

Cells $\left(2 \times 10^{5}\right)$ were starved for $24 \mathrm{~h}$ and then transferred into a 24-well Transwell with 8 um-pore membrane insert (Corning, Corning, NY, USA) after resuspension in serum-free F12 medium. The F12 medium containing $1 \%$ hormone-deficient serum and $100 \mathrm{ng} / \mathrm{mL}$ MCP-1 was used as a chemoattractant and placed in the lower chamber. After incubation for $72 \mathrm{~h}$, cells that penetrated the membrane were fixed with $95 \%$ ethanol and stained with crystal violet. Then, invaded cells were observed and calculated under a microscope.

\section{RNA isolation and RT-PCR}

Total RNA from tissues and cells was isolated using TRIzol reagent (Invitrogen, Carlsbad, CA, USA) according to the manufacturer's instructions. The ReverTra Ace qPCR RT Kit (Toyobo Co., Ltd, Kita-ku, Osaka, Japan) was used to transcribe total RNAs into cDNAs, and RT-PCR was performed using THUNDERBIRD SYBR® qPCR Mix (Toyobo Co., Ltd, Kita-ku, Osaka, Japan) with a CFX96 Touch Real-Time PCR Detection System instrument (Bio-Rad, Hercules, CA, USA). The primer sequences are listed in Table 3. The expression

Table 2. Primer sequences for luciferase reporter experiments. UTR: untranslated region

\begin{tabular}{lll}
\hline Gene & & Primer sequence \\
\hline \multirow{2}{*}{ TRAF6 3' UTR } & Sense & 5'-ATTAAGCTTAGCTGAAAACTTCTGGCTCACA-3' \\
& Antisense & 5'-CCGACGCGTCATGGGTAGCCTTCGGAGGGAG-3' \\
\multirow{2}{*}{ TRAF6 3' UTR mutation } & Sense & 5'-CTATAACAGGTTAAAAAAATGTCTTCTCAACTTTCTAGAGCAATA-3' \\
& Antisense & 5'-TATTGCTCTAGAAAGTTGAGAAGACATTTTTTTAACCTGTTATAG-3' \\
\hline
\end{tabular}




\section{Cellular Physiology Cell Physiol Biochem 2016;40:486-498 \begin{tabular}{l|l|l}
\hline DOI: 10.1159/000452563 & (c) 2016 The Author(s). Published by S. Karger AG, Basel
\end{tabular} and Biochemistry Published online: November 25, 2016 www.karger.com/cpb \\ Yin et al.: MiR-146a/TRAF6 Alters IL-17/ICAM-1 in Polymyositis}

levels of the target genes were normalized Table 3. RT-PCR primer sequences for GAPDH and miR-146a. to that of glyceraldehyde-3-phosphate GAPDH: phosphoglyceraldehyde dehydrogenase, RT-PCR: real dehydrogenase (GAPDH), and the $2^{-\Delta \Delta C t}$ time-polymerase chain reaction

method was used for calculations.

Western blotting

Tissues and cells were harvested and lysed with RIPA buffer. Total proteins were separated and calculated using the Bradford method [21]. Then, total protein was denatured in boiling water and transferred onto a polyvinylidene fluoride (PVDF) membrane after sodium dodecyl sulfate-polyacrylamide gel electrophoresis (SDS-PAGE). The membrane was blocked in TBST with $5 \%$ skim milk for $1 \mathrm{~h}$ and treated with primary antibodies against TRAF6, IL-17, ICAM-1 (1:800, 1:500, and 1:800, respectively; Zhongshan Biology Company, Beijing, China) at $4^{\circ} \mathrm{C}$ overnight. After washing, the membrane was incubated with secondary antibodies (horseradish peroxidase-conjugated goat anti-rabbit, 1:2000 dilution; Zhongshan Biology Company, Beijing, China). The samples were then imaged with enhanced chemiluminescence and quantified using the Lab Works 4.5 software with GAPDH as an endogenous control.

\section{Statistical analysis}

All statistical analyses were performed with SPSS 18.0 software (Version X; IBM, Armonk, NY, USA). Data are presented as mean \pm SD calculated from at least three repeated experiments. A two-tailed Student's $t$-test or one-way Analysis of Variance (ANOVA) was used to analyze between-group comparisons among more than two groups; $P<0.05$ was considered statistically significant.

\section{Results}

Clinical assessments, muscle histopathology and effects of conventional hormone treatment

Positive CD163 staining was detected among mononuclear inflammatory cells, particularly macrophages. No CD163 expression was observed in the muscle samples of healthy controls (Fig. 1C), both prior to treatment (Fig. 1A) and after treatment (Fig. 1B). The stained area representing macrophages produced-CD163 expression decreased after hormone treatment.

Compared with healthy controls, the muscle performance prior to treatment in the PM/DM group was much lower, but conventional hormone treatment, there was a marked improvement. Similarly, s-CK was up-regulated in patients prior to treatment compared with healthy controls and was down-regulated after hormone treatment (Fig. 2A, Table 1).

Expression of miR-146a, TRAF6, IL-17, and ICAM-1 in the muscle samples of patients and healthy controls

Compared with the control group, miR-146a expression was significantly decreased in patients both prior to treatment and after treatment, and after the conventional hormone treatment a sharp increase in miR-146a expression was observed (all $P<0.05$, Fig. 2B). TRAF6, IL-17 and ICAM-1 expression demonstrated the opposite expression trend compared

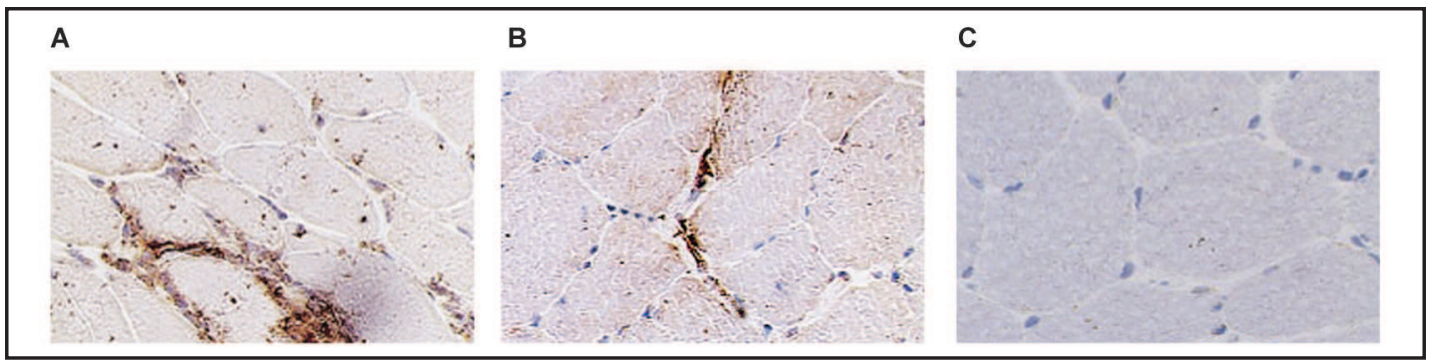

Fig. 1. Immunohistochemical staining for CD163 (stained in brown) in muscle samples from patients before treatment $(\mathrm{A})$, after treatment $(\mathrm{B})$ and healthy controls $(\mathrm{C})$. 


\section{Cellular Physiology \\ Cell Physiol Biochem 2016;40:486-498

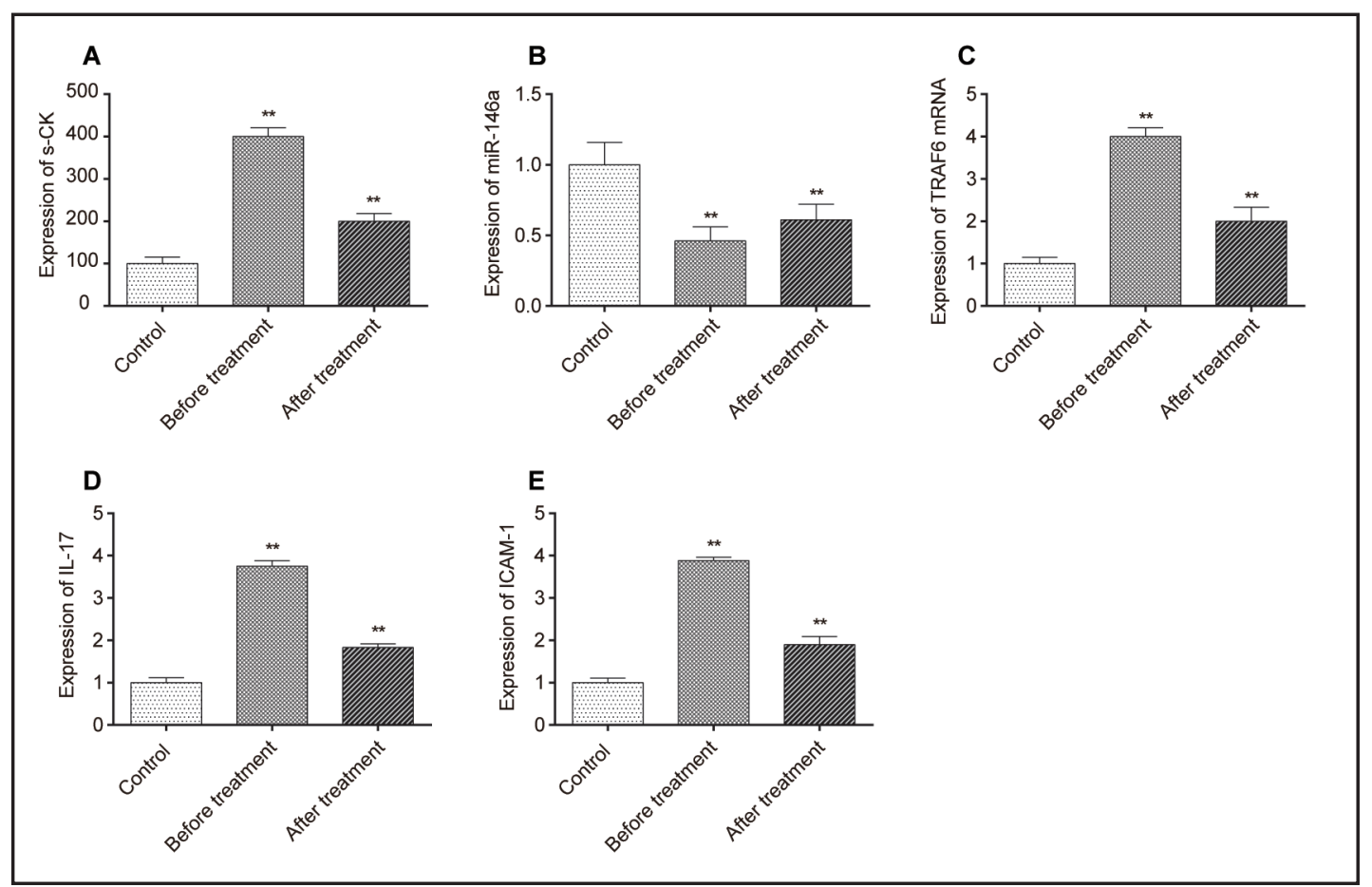

Fig. 2. Expression levels of s-CK (A), miR-146a (B), TRAF6 (C), IL-17 (D) and ICAM-1 (E) in patients before treatment, after treatment and healthy controls. Data are presented as mean \pm SD. ${ }^{* *} P<0.01$ versus patients in the before-treatment group, ${ }^{\#} P<0.05$, ${ }^{\# \#} P<0.01$ versus patients in the after-treatment group. S-CK: serum creatine phosphokinase.

Fig. 3. Immunohistochemical staining with CD163 (stained in brown) in model rats from the control (A), model (B), anti-IL-17 (C) and anti-ICAM-1 (D) groups.

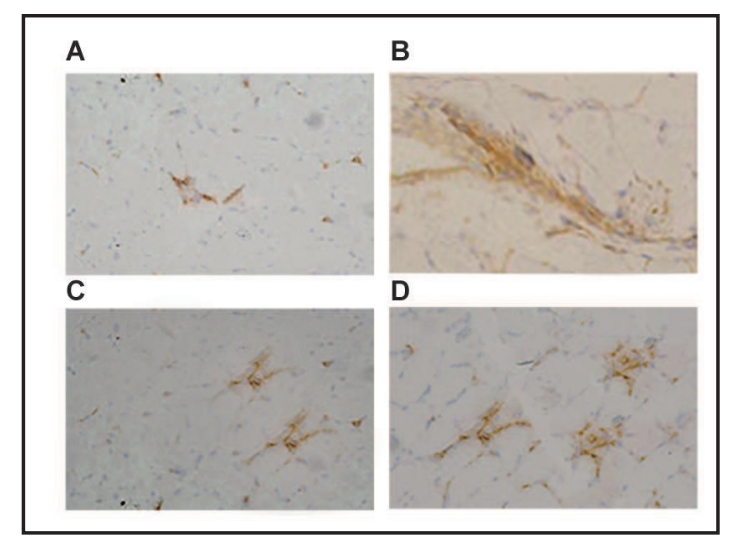

with miR-146a, i.e. patients in the control group exhibited the lowest levels, whereas the patients in the patient group prior to treatment had the highest levels of TRAF6, IL-17 and ICAM-1, and there were significant differences between the patient group prior to treatment, the patient group after treatment and the control group (all $P<0.05$, Fig. 2C-E).

Expression of CD-163, s-CK, miR-146a, TRAF6, IL-17, and ICAM-1 in the muscle samples of polymyositis model rats

Muscle samples from the control, model, anti-IL-17 and Anti-ICAM-1 groups were stained with anti-CD163 to reveal the inflammatory macrophage infiltration. CD163 was hardly observed in the control group (Fig. 3A), but demonstrated a marked increase in the model group (Fig. 3B). The treatment with anti-IL-17 or anti-ICAM-1 resulted in the pronounced down-regulation of CD163 expression (Fig. 3C-D).

As shown in Fig. 4A, the control group demonstrated the lowest s-CK expression levels, whereas the model group exhibited significantly higher s-CK expression than the control 


\section{Cellular Physiology Cell Physiol Biochem 2016;40:486-498 \begin{tabular}{ll|l} 
DOI: 10.1159/000452563 & ( 2016 The Author(s). Published by S. Karger AG, Basel
\end{tabular} \\ Yin et al.: MiR-146a/TRAF6 Alters IL-17/ICAM-1 in Polymyositis}

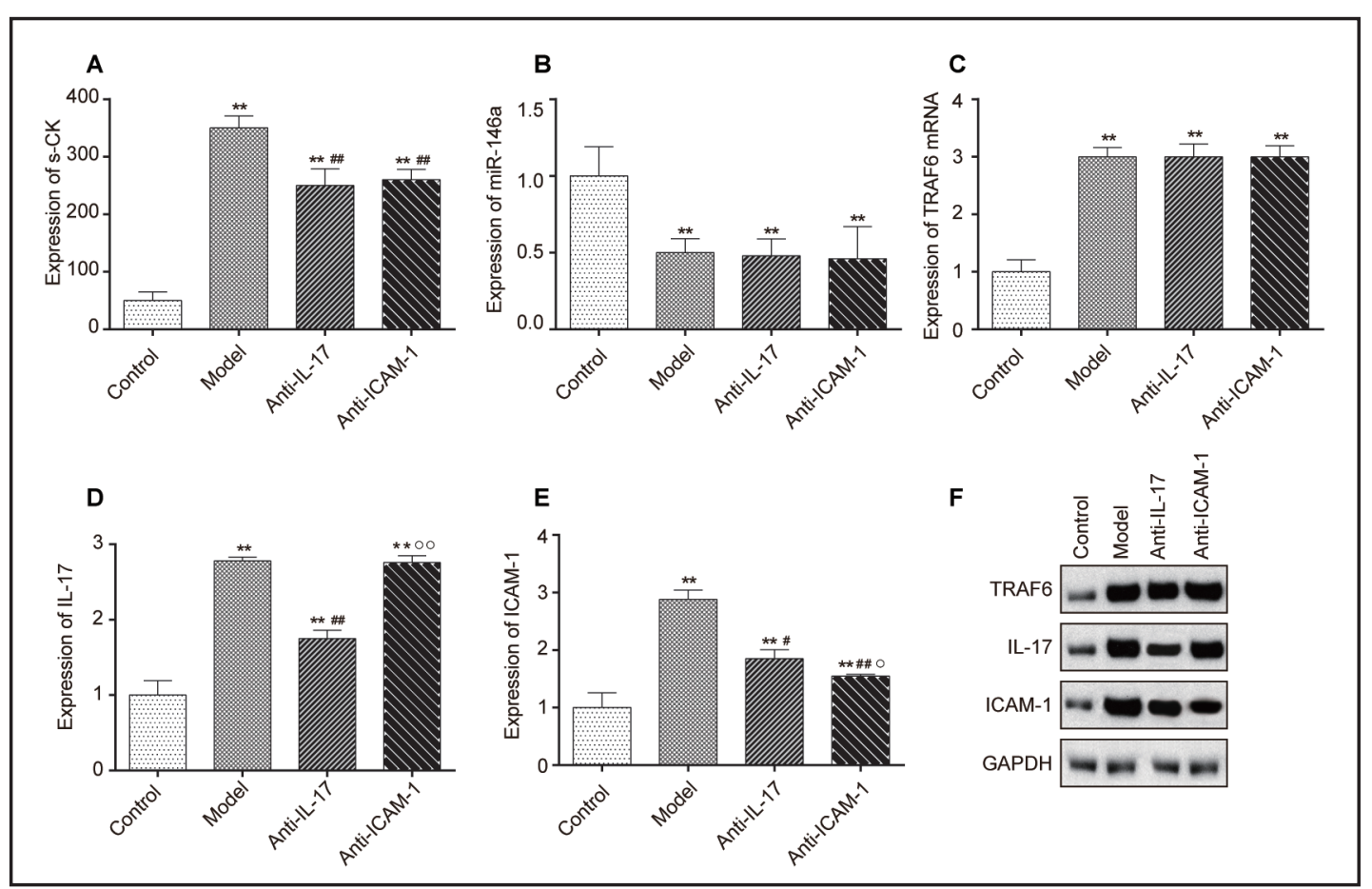

Fig. 4. Expression of s-CK, miR-146a, TRAF6, IL-17 and ICAM-1 in model rats. (A) s-CK levels in rats receiving different treatments (i.e., control, model, anti-IL-17 and anti-ICAM-1). (B) Quantitative data for the expression levels of miR-146a in model rats. (C) Quantitative data for expression levels of TRAF6 mRNA in model rats. (D-E) Quantitative protein levels of IL-17 (D) and ICAM-1 (E) in model rats. (F) Western blot analysis of IL-17 and ICAM-1 in model rats with GAPDH as an internal control. Data are presented as mean $\pm \mathrm{SD} .{ }^{* *} P<0.01$ versus the control group, ${ }^{\#} P<0.05,{ }^{\# \#} \mathrm{P}<0.01$ versus the model group, ${ }^{\circ} P<0.05,{ }^{\circ} P<0.01$ versus the anti-IL-17 group.

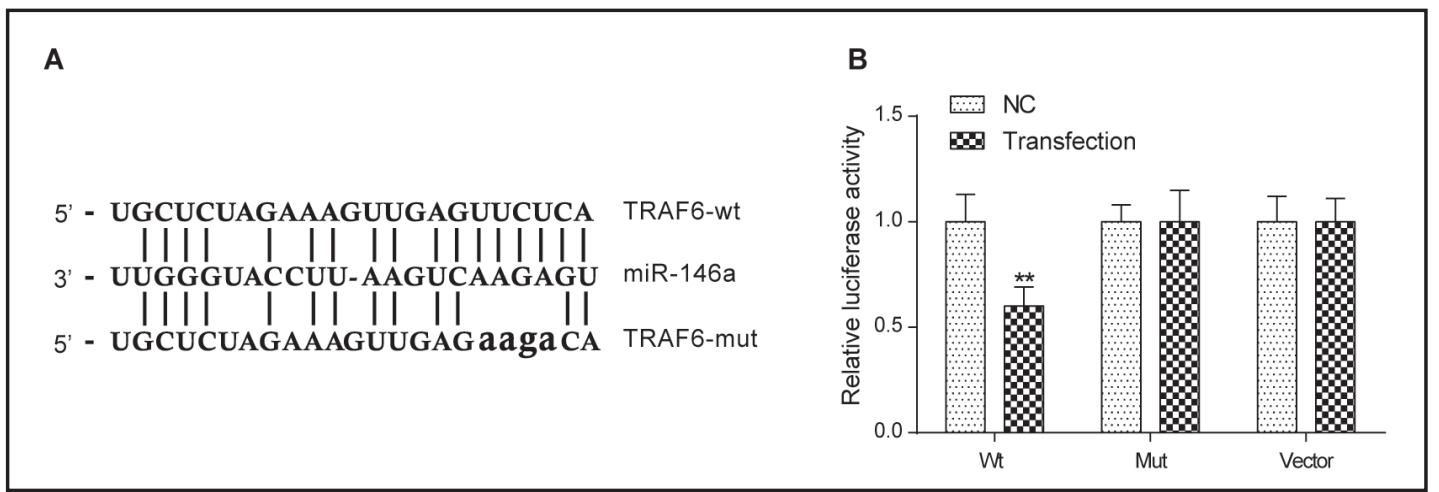

Fig. 5. Binding site prediction and luciferase assay results. (A) Putative targets predicted by TargetScan. (B) Relative luciferase activity resulted from the binding of the TRAF6 3' UTR and miR-146a in macrophages 48 h after transfection. * $P<0.05$ versus the NC group. WT: wild-type TRAF6-3' UTR; Mut: TRAF6 mut-3' UTR; NC: negative control.

group $(P<0.05)$. S-CK expression in the anti-IL-17 and anti-ICAM-1 groups was significantly lower than the model group but still higher than the control group $(P<0.05)$, whereas no significant difference was observed between the anti-IL-17 group and the anti-ICAM-1 group in terms of s-CK expression $(P>0.05)$.

As shown in Fig. 4B-F, compared with the control group, the model group demonstrated significantly higher expression of TRAF6, IL-17 and ICAM-1 but lower expression of miR- 


\section{Cellular Physiology Cell Physiol Biochem 2016;40:486-498 \begin{tabular}{ll|l} 
and Biochemist 10.1159/000452563 & $\begin{array}{l}\text { O } 2016 \text { The Author(s). Published by S. Karger AG, Basel } \\
\text { www.karger.com/cpb }\end{array}$ \\
\hline
\end{tabular} Yin et al.: MiR-146a/TRAF6 Alters IL-17/ICAM-1 in Polymyositis}

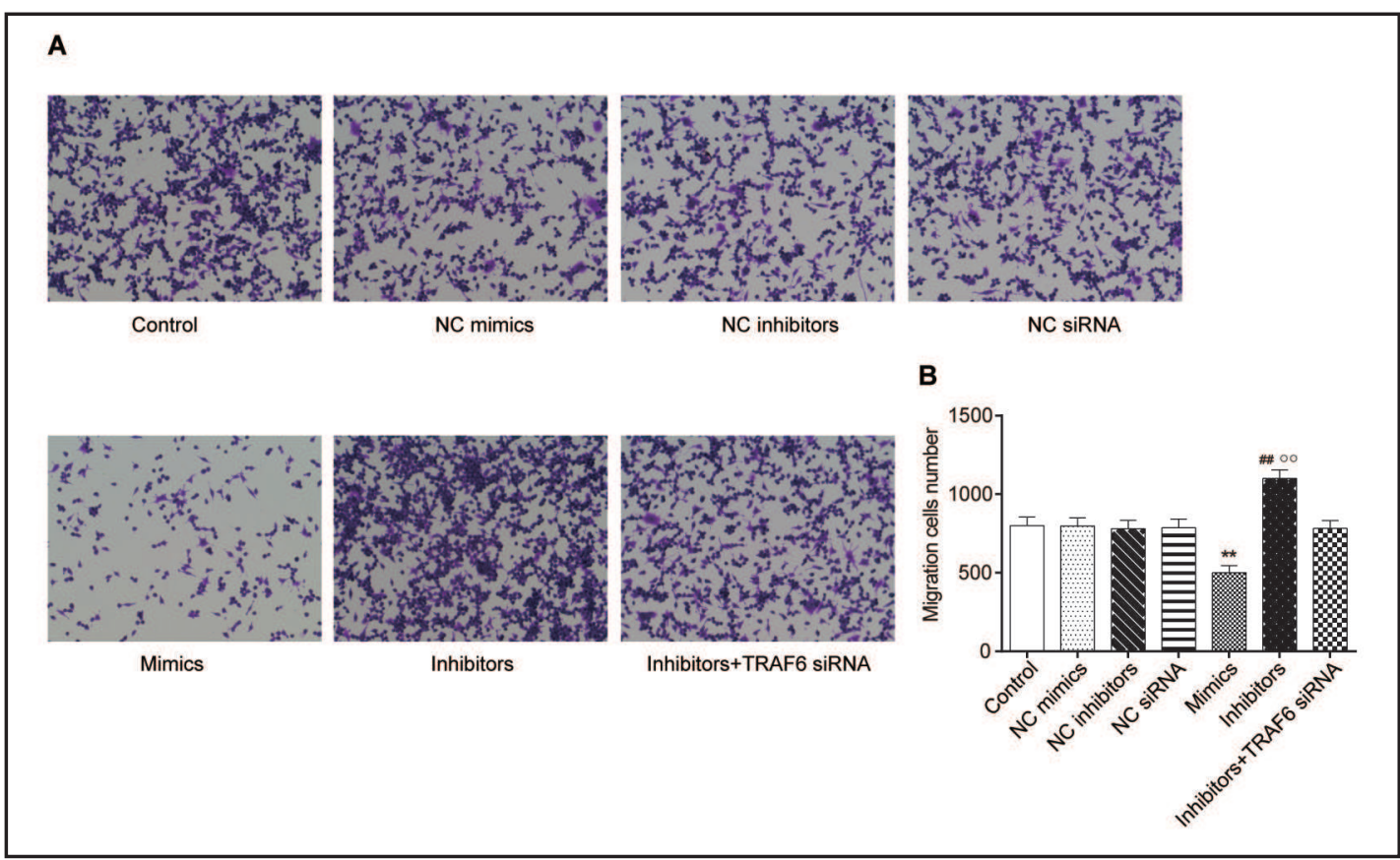

Fig. 6. MiR-146a up-regulates macrophage migration. (A) Amounts of cells that penetrated the insert membrane in the control, NC mimics, NC inhibitors, NC siRNA, miR-146a mimics, miR-146a inhibitors, miR-146a inhibitors+ TRAF6 siRNA groups. (B) Migratory cell numbers. Data are presented as mean \pm SD for three independent experiments. ${ }^{* *} P<0.01$ versus the NC mimics group, ${ }^{\# \#} P<0.01$ versus the NC inhibitors group, ${ }^{\circ} P<0.01$ versus the miR-146a mimics group.

146a (all $P<0.05$ ). Treatment with anti-IL-17 remarkably down-regulated the expression of IL-17 and ICAM-1 $(P<0.05)$, but had little effect on miR-146a expression $(P>0.05)$. Furthermore, anti-ICAM-1 treatment strongly decreased ICAM-1 expression $(P<0.05)$ but had no effect on the expression of miR-146a and IL-17 $(P>0.05)$.

MiR-146a suppresses TRAF6 expression by binding to the 3'UTR

One highly conserved miR-146a binding site was predicted in the TRAF6 3' UTR by TargetScan software (Fig. 5A). The luciferase activity assay was used to assess the direct interaction between miR-146a and TRAF6. As shown in Fig. 5B, compared with the NC group, only transfection with wt TRAF6 3' UTR showed significantly decreased relative luciferase activity $(P<0.05)$, whereas no difference was observed in the cells transfected with mut TRAF6 3' UTR and miR-146a from the corresponding control group $(P>0.05)$.

\section{MiR-146a/TRAF6 regulates macrophage migration}

The numbers of migrated cells in the Transwell assay substantially decreased after transfection with miR-146a mimics compared with the NC mimics group $(P<0.05$, Fig. 6). Treatment with miR-146a inhibitors resulted in a marked increase in migratory cell numbers compared with treatment with NC inhibitors, whereas this effect was antagonized by TRAF6 siRNA transfection $(P<0.05$, Fig. 6).

MiR-146a/TRAF6 regulate the expression of IL-17 and ICAM-1

Compared with the control group, the miR-146a mimics group demonstrated significantly higher expression levels of miR-146a. While the miR-146a inhibitors group exhibited significantly lower expression levels of miR-146a (all $P<0.05$ ), and there was no significant difference between the miR-146a inhibitors and the miR-146a inhibitors + TRAF6 siRNA group $(P>0.05$, Fig. 7A). 


\section{Cellular Physiology Cell Physiol Biochem 2016;40:486-498 \begin{tabular}{ll|l} 
DOI: 10.1159/000452563 & O 2016 The Author(s). Published by S. Karger AG, Basel \\
and Biochemistry Published online: November 25, 2016 & wwr.com/cpb
\end{tabular}

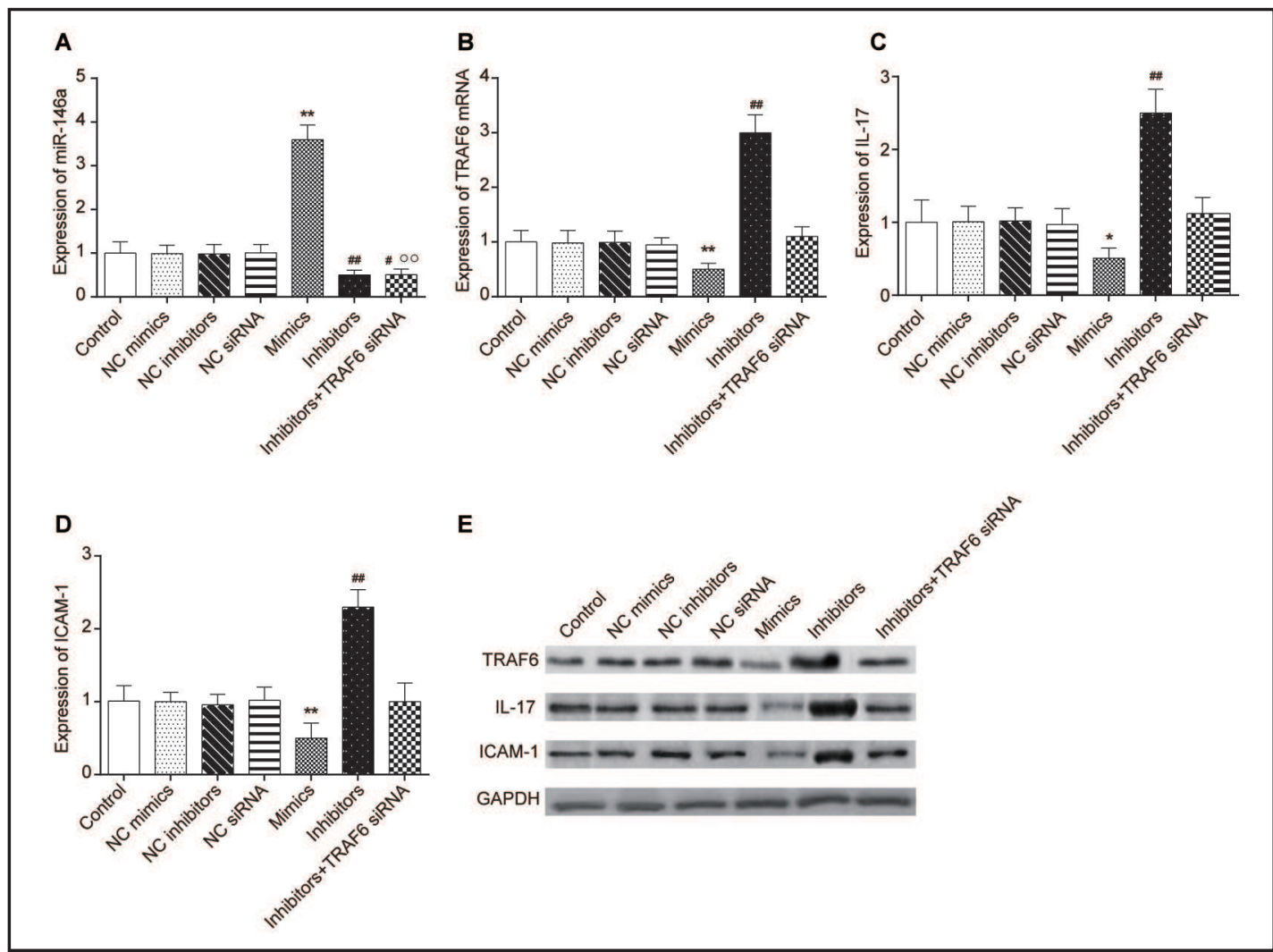

Fig. 7. MiR-146a regulates the expression of IL-17 and ICAM-1. (A) Quantitative data for miR-146a expression levels in macrophage cells. (B-D) Quantitative protein levels of TRAF6 (B), IL-17 (C) and ICAM-1 (D) in macrophage cells. E: Western blot analysis of TRAF6, IL-17 and ICAM-1 in macrophage cells with GAPDH as an internal control. Data are presented as mean \pm SD for three independent experiments. ${ }^{*} P<0.05,{ }^{* *} P<$ 0.01 versus the NC mimics group, ${ }^{\# \#} P<0.01$ versus the NC inhibitors group, ${ }^{\circ} P<0.01$ versus the NC siRNA group.

In terms of the expression of TRAF6 in transfected cells, the miR-146a mimics group exhibited significantly lower expression, whereas the miR-146a inhibitors group demonstrated significantly higher expression compared with the NC inhibitors and miR146a inhibitors + TRAF6 siRNA groups (all $P<0.05$ ).

As shown in Fig. 7C, D, E, compared with the NC mimics group, the transfection with miR-146a mimics significantly down-regulated the expression of IL-17 and ICAM-1, whereas the transfection with miR-146a inhibitors sharply up-regulated the expression of these molecules $(P<0.05)$. The expression of IL-17 and ICAM-1 in the miR-146a inhibitors + TRAF6 siRNA group was significant lower than that of the miR-146a inhibitors group but higher than that of the miR-146a mimics group $(P<0.05)$.

\section{Discussion}

As two idiopathic inflammatory myopathies (IIM), polymyositis and dermatomyositis (PM/DM) are characterized by chronic muscle weakness and inflammation in the muscle tissue accompanied by an increase in serum creatinine kinase (s-CK) levels, which leads to disability and other complications [22]. Until now, we have known little about the concrete pathogenesis of PM/DM, although several studies have demonstrated the important role of cellular inflammatory reactions in the progression of PM/DM [23, 24]. T cells and B cells are 


\section{Cellular Physiology Cell Physiol Biochem 2016;40:486-498 \begin{tabular}{ll|l} 
and BOI: 10.1159/000452563 & $\begin{array}{l}\text { C) 2016 The Author(s). Published by S. Karger AG, Basel } \\
\text { www.karger.com/cpb }\end{array}$
\end{tabular} \\ Yin et al.: MiR-146a/TRAF6 Alters IL-17/ICAM-1 in Polymyositis}

widely distributed in the skeletal muscles of PM/DM patients [25]. In addition to immune mechanisms, non-immune mechanisms are also regarded as contributing factors in the pathogenesis of PM/DM, including endoplasmic reticulum stress and autophagy in skeletal muscle cell death and dysfunction in myositis [26]. However, the specific contributions of these pathways to the PM/DM process are not completely understood. A great deal of research must be carried out before we are able to identify the mechanisms underlying PM/ DM.

In this study conducted on PM/DM patients, we observed a significant increase in IL-17 and ICAM-1 levels compared with the healthy control group. Pathogenic progress was accompanied by the exacerbation of microphage infiltration, while this inflammatory condition was alleviated by traditional hormonal therapy. Many studies have examined the relationship between IL-17, ICAM-1 and PM [27-30]. Previous studies have revealed the strong impact of IL-17 on the pathogenesis of a number of other autoimmune diseases, including psoriasis [31], rheumatoid arthritis [32] and multiple sclerosis [33], leading to the development of IL-17-targeted monoclonal antibody therapies. There are higher levels of IL17 among PM/DM patients [34, 35]. Additionally, in muscle tissue, IL-17 acts in conjunction with other pro-inflammatory cytokines produced by monocytes and innate immune responses to magnify immune responses potentially resulting in muscle destruction [36]. However, ICAM-1 use is associated with the formation of microvessels and may contribute to the activation of endothelial cells (ECs) [37]. In recent years, the important role of the vascular system in the pathogenesis of PM/DM has been revealed in several studies [38-40]. Changes in microvessels may influence the local circulation of the muscle, thus leading to the pullulation of hypoxia symptoms. Continuously, regional hypoxia in muscle tissue results in myasthenia and muscular fatigue, which are indications of early-stage PM/DM [41]. Thus, both ICAM-1 and IL-17 contribute to the deterioration of PM/DM, and their roles in this process deserve further attention.

On the contrary, in both patients and animal models, miR-146a expression levels were much lower than normal subjects or the control group, respectively. Furthermore, when mimics or inhibitors of miR-146a were experimentally applied to influence miR146a expression levels, we detected effects on IL-17 and ICAM-1 levels as well as the migratory capacity of macrophages. All of these results validated our hypothesis that miR146a regulates the expression of IL-17 and ICAM-1 in PM/DM patients. A previous study recognized the importance of miR-146a in PM/DM [42]. Although there have been few investigations examining specific regulatory mechanisms, the abnormal expression of miR146a has been verified by researchers [43]. Similarly, our study has unveiled the possible mechanism underlying the regulation of miR-146a on IL-17 and ICAM-1 expression in PM/ DM.

We were surprised to observe the positive influence of miR-146a inhibitors on IL-17 and ICAM-1 expression as well as the macrophage migration, which was counteracted by transfecting TRAF6 siRNA, consistent with our assumption that TRAF6 is involved in the regulation of miR-146a in PM/DM. MiR-146a was reported to target TRAF6 to alter the progress of type 2 diabetes mellitus (T2DM) [44], prostate cancer [45] and arthritis [46]. As one of the most vital signaling molecules in cells, TRAF6 acts directly with interleukin-1 receptor-associated kinase-1 (IRAK) and nuclear factor kappa B (NF- $\mathrm{KB}$ ) receptors to activate the signal transduction channels [47]. NF- $\kappa B$ signaling pathway is an important pathway to regulate the expression of immunoregulatory and proinflammatory mediators [48], and the innate immune and inflammatory responses play critical roles during the pathogenesis of PM/DM [45]. Thus, we believe that miR-146a targets TRAF6 to regulate the pathogenesis of $\mathrm{PM} / \mathrm{DM}$, an observation that has not been reported in any other studies. In addition, miR146a targets TRAF6 and influences the production of IL-17 in psoriatic patients [49]. ICAM1 expression has been linked to this signaling pathway in certain chronic inflammatory diseases such as diabetic retina [50]. Our study demonstrated the effects of miR-146a/ TRAF 6 axis on the expression of IL-17 and ICAM-1, which then influence the pathogenesis of PM/DM. 


\section{Cellular Physiology Cell Physiol Biochem 2016;40:486-498 \begin{tabular}{l|l|l}
\cline { 2 - 3 } DOI: 10.1159/000452563 & (c) 2016 The Author(s). Published by S. Karger AG, Basel
\end{tabular} and Biochemistry Published online: November 25, 2016 www.karger.com/cpb \\ Yin et al:: MiR-146a/TRAF6 Alters IL-17/ICAM-1 in Polymyositis}

As was mentioned, the NF- $\kappa \mathrm{B}$ signaling pathway arose our interest and its role in PM/ DM development requires further investigation. We intended to conduct our further studies to study the possible interplay between the miR-146a/TRAF6 axis and the signaling pathway.

In summary, for the first time, this study demonstrates the ability of TRAF6 targeted by miR-146a to regulate the expression of IL-17 and ICAM-1. This discovery will contribute to our understanding of the development of PM/DM pathogenesis and facilitate diagnosis. Further study is required to elucidate the underlying mechanism and other regulatory factors as well as the roles of NF- $\mathrm{KB}$ signaling and targeting proteins.

\section{Disclosure Statement}

The authors declare no commercial or financial conflicts of interest.

\section{References}

1 Mammen AL: Dermatomyositis and polymyositis: Clinical presentation, autoantibodies, and pathogenesis. Ann N Y Acad Sci 2010;1184:134-153.

2 Findlay AR, Goyal NA, Mozaffar T: An overview of polymyositis and dermatomyositis. Muscle Nerve 2015;51:638-656.

3 Dalakas MC: Immunotherapy of myositis: issues, concerns and future prospects. Nat Rev Rheumatol 2010;6:129-137.

4 Milisenda JC, Selva-O'Callaghan A, Grau JM: The diagnosis and classification of polymyositis. J Autoimmun 2014;48-49:118-121.

5 Niimoto T, Nakasa T, Ishikawa M, Okuhara A, Izumi B, Deie M, Suzuki O, Adachi N, Ochi M: MicroRNA-146a expresses in interleukin-17 producing T cells in rheumatoid arthritis patients. BMC Musculoskelet Disord 2010;11:209.

6 Nakae S, Komiyama Y, Nambu A, Sudo K, Iwase M, Homma I, Sekikawa K, Asano M, Iwakura Y: Antigenspecific T cell sensitization is impaired in IL-17-deficient mice, causing suppression of allergic cellular and humoral responses. Immunity 2002;17:375-387.

7 Matusevicius D, Kivisakk P, He B, Kostulas N, Ozenci V, Fredrikson S, Link H: Interleukin-17 mRNA expression in blood and CSF mononuclear cells is augmented in multiple sclerosis. Mult Scler 1999;5:101104.

8 Wong CK, Ho CY, Li EK, Lam CW: Elevation of proinflammatory cytokine (IL-18, IL-17, IL-12) and Th2 cytokine (IL-4) concentrations in patients with systemic lupus erythematosus. Lupus 2000;9:589-593.

9 Holland J, Owens T: Signaling through intercellular adhesion molecule 1 (ICAM-1) in a B cell lymphoma line. The activation of Lyn tyrosine kinase and the mitogen-activated protein kinase pathway. J Biol Chem 1997;272:9108-9112.

10 Othumpangat S, Noti JD, McMillen CM, Beezhold DH: ICAM-1 regulates the survival of influenza virus in lung epithelial cells during the early stages of infection. Virology 2016;487:85-94.

11 Pietruczuk M, Pietruczuk A, Pancewicz S, Hermanowska-Szpakowicz T: [ICAM-1: structure, biological role and clinical significance]. Pol Merkur Lekarski 2004;17:507-511.

12 Gabr MA, Jing L, Helbling AR, Sinclair SM, Allen KD, Shamji MF, Richardson WJ, Fitch RD, Setton LA, Chen J: Interleukin-17 synergizes with IFNgamma or TNFalpha to promote inflammatory mediator release and intercellular adhesion molecule-1 (ICAM-1) expression in human intervertebral disc cells. J Orthop Res 2011;29:1-7.

13 Eisenberg I, Eran A, Nishino I, Moggio M, Lamperti C, Amato AA, Lidov HG, Kang PB, North KN, MitraniRosenbaum S, Flanigan KM, Neely LA, Whitney D, Beggs AH, Kohane IS, Kunkel LM: Distinctive patterns of microRNA expression in primary muscular disorders. Proc Natl Acad Sci USA 2007;104:17016-17021.

14 Ambros V: The functions of animal microRNAs. Nature 2004;431:350-355. 


\section{Cellular Physiology Cell Physiol Biochem 2016;40:486-498 \begin{tabular}{ll|l} 
DOI: 10.1159/000452563 & $\begin{array}{l}\text { O 2016 The Author(s). Published by S. Karger AG, Basel } \\
\text { www.karger.com/cpb }\end{array}$ \\
\hline
\end{tabular} \\ Yin et al.: MiR-146a/TRAF6 Alters IL-17/ICAM-1 in Polymyositis}

15 Lofgren SE, Frostegard J, Truedsson L, Pons-Estel BA, D'Alfonso S, Witte T, Lauwerys BR, Endreffy E, Kovacs L, Vasconcelos C, Martins da Silva B, Kozyrev SV, Alarcon-Riquelme ME: Genetic association of miRNA-146a with systemic lupus erythematosus in Europeans through decreased expression of the gene. Genes Immun 2012;13:268-274.

16 Boldin MP, Taganov KD, Rao DS, Yang L, Zhao JL, Kalwani M, Garcia-Flores Y, Luong M, Devrekanli A, Xu J, Sun G, Tay J, Linsley PS, Baltimore D: miR-146a is a significant brake on autoimmunity, myeloproliferation, and cancer in mice. J Exp Med 2011;208:1189-1201.

17 Yang CR, Shih KS, Liou JP, Wu YW, Hsieh IN, Lee HY, Lin TC, Wang JH: Denbinobin upregulates miR146a expression and attenuates IL-1beta-induced upregulation of ICAM-1 and VCAM-1 expressions in osteoarthritis fibroblast-like synoviocytes. J Mol Med (Berl) 2014;92:1147-1158.

18 Troyanov Y, Targoff IN, Payette MP, Raynauld JP, Chartier S, Goulet JR, Bourre-Tessier J, Rich E, Grodzicky T, Fritzler MJ, Joyal F, Koenig M, Senecal JL: Redefining dermatomyositis: a description of new diagnostic criteria that differentiate pure dermatomyositis from overlap myositis with dermatomyositis features. Medicine (Baltimore) 2014;93:318-332.

19 Henriksson KG: "Semi-open" muscle biopsy technique. A simple outpatient procedure. Acta Neurol Scand 1979;59:317-323.

20 Han LN, Li TL, Ding GL, Liu JW, Ding Y, Zhang YJ: [Establishment of human cardiac C protein induced experimental autoimmune myocarditis model in rat]. Zhonghua Xin Xue Guan Bing Za Zhi 2012;40:690696.

21 Kruger NJ: The Bradford method for protein quantitation. Methods Mol Biol 1994;32:9-15.

22 Pandya JM, Loell I, Hossain MS, Zong M, Alexanderson H, Raghavan S, Lundberg IE, Malmstrom V: Effects of conventional immunosuppressive treatment on CD244+ (CD28null) and FOXP3+ T cells in the inflamed muscle of patients with polymyositis and dermatomyositis. Arthritis Res Ther 2015;18:80.

23 Notarnicola A, Lapadula G, Natuzzi D, Lundberg IE, Iannone F: Correlation between serum levels of IL-15 and IL-17 in patients with idiopathic inflammatory myopathies. Scand J Rheumatol 2015;44:224-228.

24 Salomonsson S, Lundberg IE: Cytokines in idiopathic inflammatory myopathies. Autoimmunity 2006;39:177-190.

25 Rayavarapu S, Coley W, Nagaraju K: An update on pathogenic mechanisms of inflammatory myopathies. Curr Opin Rheumatol 2011;23:579-584.

26 Henriques-Pons A, Nagaraju K: Nonimmune mechanisms of muscle damage in myositis: role of the endoplasmic reticulum stress response and autophagy in the disease pathogenesis. Curr Opin Rheumatol 2009;21:581-587.

27 Szodoray P, Alex P, Knowlton N, Centola M, Dozmorov I, Csipo I, Nagy AT, Constantin T, Ponyi A, Nakken B, Danko K: Idiopathic inflammatory myopathies, signified by distinctive peripheral cytokines, chemokines and the TNF family members B-cell activating factor and a proliferation inducing ligand. Rheumatology (Oxford) 2010;49:1867-1877.

28 Tournadre A, Porcherot M, Cherin P, Marie I, Hachulla E, Miossec P: Th1 and Th17 balance in inflammatory myopathies: interaction with dendritic cells and possible link with response to high-dose immunoglobulins. Cytokine 2009;46:297-301.

29 Wang M, Jia JP, Da YW: [A study of vascular factor in the pathogenesis of polymyositis]. Zhonghua Nei Ke Za Zhi 2005;44:115-117.

30 Sallum AM, Kiss MH, Silva CA, Wakamatsu A, Vianna MA, Sachetti S, Marie SK: Difference in adhesion molecule expression (ICAM-1 and VCAM-1) in juvenile and adult dermatomyositis, polymyositis and inclusion body myositis. Autoimmun Rev 2006;5:93-100.

31 Baeten DL, Kuchroo VK: How Cytokine networks fuel inflammation: Interleukin-17 and a tale of two autoimmune diseases. Nat Med 2013;19:824-825.

32 Kenna TJ, Brown MA: The role of IL-17-secreting mast cells in inflammatory joint disease. Nat Rev Rheumatol 2013;9:375-379.

33 Miossec P: Interleukin-17 in fashion, at last: ten years after its description, its cellular source has been identified. Arthritis Rheum 2007;56:2111-2115.

34 Mielnik P, Chwalinska-Sadowska H, Wiesik-Szewczyk E, Maslinski W, Olesinska M: Serum concentration of interleukin 15, interleukin 2 receptor and TNF receptor in patients with polymyositis and dermatomyositis: correlation to disease activity. Rheumatol Int 2012;32:639-643. 


\section{Cellular Physiology Cell Physiol Biochem 2016;40:486-498 \begin{tabular}{ll|l} 
DOI: 10.1159/000452563 & $\begin{array}{l}\text { ○ 2016 The Author(s). Published by S. Karger AG, Basel } \\
\text { www.karger.com/cpb }\end{array}$ \\
\hline
\end{tabular} \\ Yin et al.: MiR-146a/TRAF6 Alters IL-17/ICAM-1 in Polymyositis}

35 Zong M, Loell I, Lindroos E, Nader GA, Alexanderson H, Hallengren CS, Borg K, Arnardottir S, McInnes IB, Lundberg IE: Effects of immunosuppressive treatment on interleukin-15 and interleukin-15 receptor alpha expression in muscle tissue of patients with polymyositis or dermatomyositis. Ann Rheum Dis 2012;71:1055-1063.

36 Tournadre A, Miossec P: Interleukin-17 in inflammatory myopathies. Curr Rheumatol Rep 2012;14:252256.

37 Barbasso Helmers S, Englund P, Engstrom M, Ahlin E, Fathi M, Janciauskiene S, Heimburger M, Ronnelid J, Lundberg IE: Sera from anti-Jo-1-positive patients with polymyositis and interstitial lung disease induce expression of intercellular adhesion molecule 1 in human lung endothelial cells. Arthritis Rheum 2009;60:2524-2530.

38 Figarella-Branger D, Schleinitz N, Boutiere-Albanese B, Camoin L, Bardin N, Guis S, Pouget J, Cognet C, Pellissier JF, Dignat-George F: Platelet-endothelial cell adhesion molecule-1 and CD146: soluble levels and in situ expression of cellular adhesion molecules implicated in the cohesion of endothelial cells in idiopathic inflammatory myopathies. J Rheumatol 2006;33:1623-1630.

39 Volpi N, Pecorelli A, Lorenzoni P, Di Lazzaro F, Belmonte G, Agliano M, Cantarini L, Giannini F, Grasso G, Valacchi G: Antiangiogenic VEGF isoform in inflammatory myopathies. Mediators Inflamm 2013;2013:219313.

40 Loenneke JP, Thiebaud RS, Abe T, Manfro IG, Marin PJ: Acute blood flow restricted exercise to treat Duchenne muscular dystrophy: would it be efficacious? Front Physiol 2013;4:114.

41 Jain A, Sharma MC, Sarkar C, Bhatia R, Singh S, Handa R: Increased expression of cell adhesion molecules in inflammatory myopathies: diagnostic utility and pathogenetic insights. Folia Neuropathol 2009;47:33-42.

42 Okada Y, Jinnin M, Makino T, Kajihara I, Makino K, Honda N, Nakayama W, Inoue K, Fukushima S, Ihn H: MIRSNP rs2910164 of miR-146a is associated with the muscle involvement in polymyositis/ dermatomyositis. Int J Dermatol 2014;53:300-304.

43 Parkes JE, Day PJ, Chinoy H, Lamb JA: The role of microRNAs in the idiopathic inflammatory myopathies. Curr Opin Rheumatol 2015;27:608-615.

44 Lenin R, Sankaramoorthy A, Mohan V, Balasubramanyam M: Altered immunometabolism at the interface of increased endoplasmic reticulum (ER) stress in patients with type 2 diabetes. J Leukoc Biol 2015;98:615622.

45 Liu R, Yi B, Wei S, Yang WH, Hart KM, Chauhan P, Zhang W, Mao X, Liu X, Liu CG, Wang L: FOXP3-miR-146NF-kappaB Axis and Therapy for Precancerous Lesions in Prostate. Cancer Res 2015;75:1714-1724.

46 Wang JH, Shih KS, Wu YW, Wang AW, Yang CR: Histone deacetylase inhibitors increase microRNA-146a expression and enhance negative regulation of interleukin-1beta signaling in osteoarthritis fibroblast-like synoviocytes. Osteoarthritis Cartilage 2013;21:1987-1996.

47 Gao M, Wang X, Zhang X, Ha T, Ma H, Liu L, Kalbfleisch JH, Gao X, Kao RL, Williams DL, Li C: Attenuation of Cardiac Dysfunction in Polymicrobial Sepsis by MicroRNA-146a Is Mediated via Targeting of IRAK1 and TRAF6 Expression. J Immunol 2015;195:672-682.

48 Zou L, Feng Y, Chen YJ, Si R, Shen S, Zhou Q, Ichinose F, Scherrer-Crosbie M, Chao W: Toll-like receptor 2 plays a critical role in cardiac dysfunction during polymicrobial sepsis. Crit Care Med 2010;38:1335-1342.

49 Xia P, Fang X, Zhang ZH, Huang Q Yan KX, Kang KF, Han L, Zheng ZZ: Dysregulation of miRNA146a versus IRAK1 induces IL-17 persistence in the psoriatic skin lesions. Immunol Lett 2012;148:151-162.

50 Wang Q Bozack SN, Yan Y, Boulton ME, Grant MB, Busik JV: Regulation of retinal inflammation by rhythmic expression of MiR-146a in diabetic retina. Invest Ophthalmol Vis Sci 2014;55:3986-3994. 